\title{
Insuffisance respiratoire aiguë : l'ARDS et au-delà
}

\section{Acute Respiratory Failure: The ARDS and Beyond}

\author{
I. Bendib - F. Schlemmer - B. Maitre - G. Carteaux $\cdot$ K. Razazi $\cdot$ A. Mekontso Dessap $\cdot$ N. de Prost
}

Reçu le 27 septembre 2017; accepté le 15 décembre 2017

(C) SRLF et Lavoisier SAS 2018

Résumé Le syndrome de détresse respiratoire aiguë (SDRA) correspond à la forme la plus grave de l'insuffisance respiratoire aiguë hypoxémique et est associé à une mortalité hospitalière de l'ordre de $40 \%$. Il s'agit d'une entité hétérogène dont la définition clinicoradiologique a été revue en 2012 lors de la conférence de Berlin. Le substrat histologique le plus fréquemment associé au SDRA est le dommage alvéolaire diffus (DAD), mais un large panel de lésions histologiques a été rapporté, suggérant que le SDRA ne peut être réduit au DAD. Des facteurs de risque « communs » ont été proposés par la conférence de Berlin et sont retrouvés dans plus de $90 \%$ des cas. Il existe cependant d'authentiques SDRA pour lesquels aucun facteur de risque n'est identifié et pouvant nécessiter une approche thérapeutique ciblée. Une démarche diagnostique standardisée est alors indispensable.

Mots clés Syndrome de détresse respiratoire aiguë -

Définition de Berlin · Dommage alvéolaire diffus · Edème pulmonaire $\cdot$ Diagnostic

Abstract The acute respiratory distress syndrome (ARDS) is
the most severe form of acute hypoxemic respiratory failure

I. Bendib · G. Carteaux · K. Razazi · A. Mekontso Dessap

N. de Prost $(\square)$

Service de réanimation médicale,

Assistance publique-Hôpitaux de Paris,

hôpitaux universitaires Henri-Mondor, DHU A-TVB, Créteil,

51, avenue du Maréchal-de-Lattre-de-Tassigny,

F-94010 Créteil, France,

et groupe de recherche clinique CARMAS,

faculté de médecine de Créteil,

université Paris-Est-Créteil, F-94010 Créteil, France

e-mail : nicolas.de-prost@aphp.fr

F. Schlemmer · B. Maitre

Assistance publique-Hôpitaux de Paris,

hôpitaux universitaires Henri-Mondor,

DHU A-TVB, antenne de pneumologie,

service de réanimation médicale, F-94010 Créteil, France,

et groupe de recherche clinique CARMAS,

faculté de médecine de Créteil,

université Paris-Est-Créteil, F-94010 Créteil, France and is associated with a hospital mortality rate of $40 \%$. The Berlin definition of ARDS was proposed in 2012 and relies on clinical and radiological features. Previous studies have linked ARDS to histological diffuse alveolar damage (DAD) but other histological patterns have been reported, suggesting that DAD is not the gold standard for ARDS. ARDS "common" risk factors have been proposed by the Berlin definition and were identified upon ARDS diagnosis in more than $90 \%$ of cases. When no ARDS risk factor is identified, a standardized diagnostic work-up is warranted in order to individualize patient management.

Keywords Respiratory distress syndrome $\cdot$ Adult $\cdot$ Berlin definition $\cdot$ Diffuse alveolar damage $\cdot$ Pulmonary edema Diagnosis

\section{Introduction}

Le syndrome de détresse respiratoire aiguë (SDRA) correspond à la forme la plus grave de l'insuffisance respiratoire aiguë hypoxémique. Dans l'étude observationnelle Lung Safe, menée dans 450 services de réanimation de 50 pays différents, le SDRA représentait $10,4 \%$ des admissions en unité de soins intensifs et $23,4 \%$ des patients nécessitant un support ventilatoire [1]. La mortalité en réanimation et hospitalière du SDRA était respectivement de 35 et $40 \%$ [1]. Sur le plan physiopathologique, le SDRA est caractérisé par une réaction inflammatoire diffuse du poumon, secondaire à une agression pulmonaire directe ou indirecte, associée à une augmentation de la perméabilité microvasculaire [2]. Des lésions pulmonaires induites par la ventilation mécanique contribuent ensuite à amplifier l'agression pulmonaire initiale $[3,4]$. Il en résulte la constitution d'un œdème pulmonaire lésionnel (augmentation du poids et perte d'aération du poumon) responsable d'un shunt intrapulmonaire, d'une augmentation de l'espace mort et d'une diminution de la compliance pulmonaire. Plusieurs définitions clinicoradiologiques du SDRA ont été proposées depuis sa description 
initiale, mais, sur le plan histologique, le SDRA a été d'emblée rattaché au dommage alvéolaire diffus (DAD) [5]. Cependant, les études histologiques réalisées au cours des deux dernières décennies ont rapporté qu'une proportion significative des patients atteints de SDRA clinicoradiologique n'avait pas de lésions histologiques de DAD $[6,7]$. L'objectif de cette revue générale est de présenter de manière synthétique les causes principales du SDRA, classiquement associées à la présence de DAD histologique, ainsi que les causes plus rares, souvent associées à d'autres lésions histologiques. Les éléments clés de la stratégie diagnostique seront également présentés. Cette revue n'a par contre pas pour objectif d'aborder les aspects liés à la prise en charge symptomatique du SDRA.

\section{Dommage alvéolaire diffus, substrat histopathologique historique du SDRA}

La description initiale du SDRA remonte à 1967. Dans un article publié dans le Lancet, Ashbaugh et al. [8] rapportaient une série de 12 patients adultes — dont 7 décédaient à la phase aiguë — pris en charge pour un tableau d'insuffisance respiratoire aiguë hypoxémique ne répondant pas à l'assistance respiratoire. Ces patients ont été considérés comme atteints d'une maladie superposable à celle des nouveau-nés prématurés, déficitaires en surfactant (maladie des membranes hyalines). Le tableau clinique de ces patients associait dyspnée sévère, tachypnée, cyanose réfractaire à l'oxygénothérapie, perte de compliance du système respiratoire et infiltrats alvéolaires diffus à la radiographie thoracique [8]. Des facteurs favorisants (traumatisme sévère, pneumonie virale, pancréatite) étaient décrits. Les sept patients décédés ont été autopsiés : les poumons étaient macroscopiquement lourds, d'aspect violacé et hépatisés — c'est à dire totalement non aérés - à la tranche de section. L'examen microscopique des poumons retrouvait un engorgement et une dilatation des capillaires, des atélectasies alvéolaires, de l'hémorragie interstitielle et alvéolaire, un œdème diffus et la présence de « membranes hyalines" correspondant à l'accumulation de débris cellulaires et de protéines sur les parois alvéolaires [8]. L'inflammation diffuse et la fibrose n'étaient observées que dans les cas de décès tardif. Quelques années plus tard, Katzenstein et al. [5] employaient le terme de dommage alvéolaire diffus (DAD) pour décrire cet ensemble de lésions histologiques caractéristiques de l'agression pulmonaire aiguë.

Les lésions de DAD sont en fait évolutives, avec trois phases successives typiquement décrites, pouvant coexister dans un même poumon : exsudative, proliférative et de fibroprolifération $[5,9]$. La phase exsudative - ou « DAD aigu » — correspond aux lésions décrites ci-dessus et prédomine dans les sept jours suivant l'agression pulmonaire. La seconde phase, proliférative, correspond à la phase de réparation alvéolaire ; elle est caractérisée par une prolifération cellulaire intense des fibroblastes et des pneumocytes de type II. Cette phase atteint son paroxysme durant la deuxième et la troisième semaine et peut aboutir à une réparation ad integrum du parenchyme pulmonaire, ou évoluer vers une fibrose persistante [5]. Dans une étude autopsique chez des patients décédés ayant les critères radiocliniques de SDRA au moment du décès, Thille et al. [10] ont étudié la cinétique de survenue de ces lésions en fonction du délai écoulé entre la survenue du SDRA et le décès (Fig. 1). Les auteurs ont ainsi démontré que les différentes phases pouvaient se chevaucher dans le temps et apparaître de façon fluctuante [10]. Comme attendu, la phase exsudative prédominait durant la première semaine, après le début de l'agression pulmonaire, mais la phase proliférative pouvait apparaître elle aussi dès la première semaine pour devenir quasi constante après trois semaines d'évolution du SDRA. Enfin, et de manière frappante, la phase de fibroprolifération était parfois observée dès le septième jour et était présente dans plus de $60 \%$ des cas au-delà de la troisième semaine [10].

\section{Définitions clinicoradiologiques du SDRA}

La première définition du SDRA a été proposée par la Conférence de consensus américano-européenne (AECC) sur le SDRA en 1994 [11] (Tableau 1). L'agression pulmonaire aiguë ou acute lung injury (ALI) était définie par l'association de signes cliniques (début aigu, $\mathrm{PaO}_{2} / \mathrm{FiO}_{2} \leq 300 \mathrm{mmHg}$ ), radiologiques (infiltrats pulmonaires bilatéraux) et un mécanisme de constitution de l'œè̀me pulmonaire non hydrostatique (absence d'élévation des pressions de remplissage du cœur gauche). Le SDRA correspondait à un sous-groupe d'ALI ayant une hypoxémie plus sévère $\left(\mathrm{PaO}_{2} / \mathrm{FiO}_{2} \leq\right.$ $200 \mathrm{mmHg}$ ) et une mortalité plus élevée. L'objectif principal de cette définition était de faciliter l'identification des patients atteints de SDRA, afin de permettre l'inclusion prospective de patients ayant une présentation clinique homogène dans des protocoles de recherche clinique, de façon à obtenir une comparabilité entre les différentes études portant sur le SDRA. Cette définition de l'AECC [11] a ainsi été largement adoptée par les cliniciens et les investigateurs et a abouti à la réalisation de nombreuses études ayant permis une amélioration des connaissances épidémiologiques, cliniques, et de la prise en charge du SDRA, l'étude ARMA étant la plus emblématique d'entre elles [4]. Cependant, après plusieurs années d'utilisation, cette définition a présenté de nombreuses limites, notamment : l'imprécision du critère de délai définissant l'apparition aiguë du tableau respiratoire, un critère d'oxygénation (rapport $\mathrm{PaO}_{2} / \mathrm{FiO}_{2}$ ) obtenu sans préciser les conditions de pression télé-expiratoire positive, un critère radiologique imprécis 


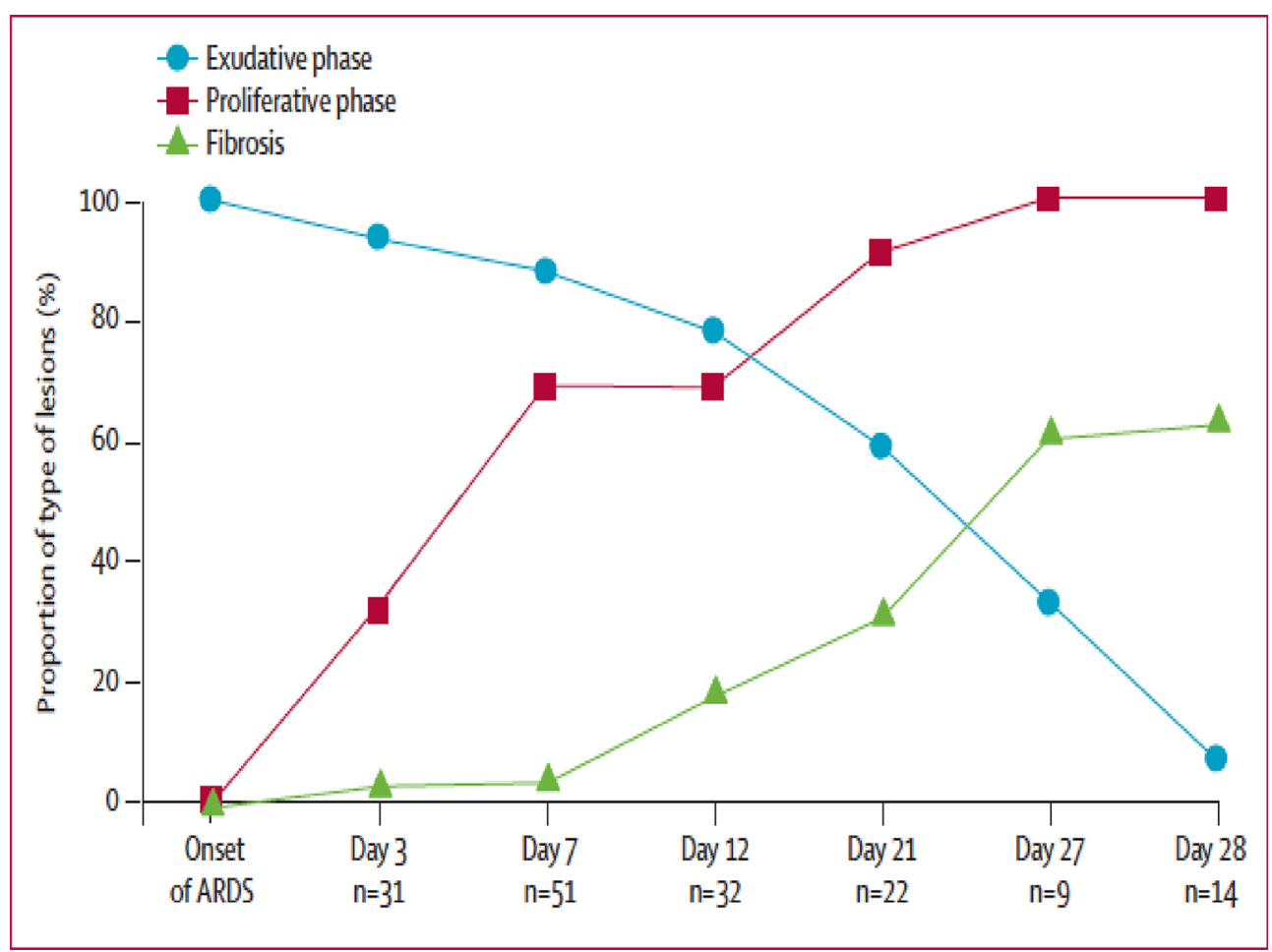

Fig. 1 Relation entre les différents stades du dommage alvéolaire diffus et le délai de survenue du SDRA chez des patients décédés avec les critères de SDRA (définition de Berlin [2]) et autopsiés. Les trois phases caractéristiques du dommage alvéolaire diffus sont représentées : en bleu, la phase exsudative ; en rouge, la phase proliférative ; en vert, la phase de fibroprolifération (d'après Thille et al. [10], avec autorisation)

et l'exclusion systématique des patients ayant un œdème pulmonaire mixte, lésionnel et hydrostatique (Tableau 1) [2].

Les performances diagnostiques de la définition de l'AECC — c'est-à-dire la corrélation avec la présence de DAD histologique - ont été étudiées en 2004 par Esteban et al. : 127 patients décédés en réanimation et remplissant les critères de SDRA au moment du décès ont été autopsiés [7]. Les patients étaient catégorisés comme ayant des lésions de DAD lorsque l'examen histologique mettait en évidence la présence de membranes hyalines associées à au moins l'une des lésions suivantes : nécrose des cellules endothéliales ou des cellules épithéliales alvéolaires de type I, œdème, fibrose interstitielle organisée ou prolifération des cellules épithéliales alvéolaires de type II [5,9]. La sensibilité et la spécificité de la définition clinique de l'AECC étaient respectivement de 75 et $84 \%$.

Pour l'ensemble des raisons évoquées ci-dessus, la définition du SDRA a été revue lors de la conférence de Berlin en 2012 [2] et comporte désormais trois catégories, selon le critère d'oxygénation obtenu avec une pression expiratoire positive de $5 \mathrm{cmH}_{2} \mathrm{O}$ (Tableau 1) : SDRA léger (rapport $\mathrm{PaO}_{2} / \mathrm{FiO}_{2}$ entre 200 et $300 \mathrm{mmHg}$ ou patient ayant un support par pression positive continue), modéré (rapport $\mathrm{PaO}_{2} / \mathrm{FiO}_{2}$ entre 100 et $200 \mathrm{mmHg}$ ) et sévère (rapport $\mathrm{PaO}_{2} / \mathrm{FiO}_{2}<100 \mathrm{mmHg}$ ). L'objectif de cette classifi- cation était de séparer, dès l'apparition des critères de SDRA, les patients en fonction de leur mortalité prédite et de proposer une adaptation de la prise en charge en fonction de leur sévérité [12]. Le délai de survenue du tableau respiratoire était également précisé et devait être au maximum de sept jours à partir de l'exposition à un ou plusieurs facteurs de risque définis. Enfin, l'œdème pulmonaire peut avoir une origine en partie hydrostatique, sauf lorsqu'aucun facteur de risque de SDRA n'est mis en évidence. Une évaluation objective des pressions de remplissage du cœur gauche devient nécessaire dans ce cas [2].

\section{Données histologiques : SDRA avec ou sans DAD}

Comme pour la définition de l'AECC, la validité de la définition de Berlin [2] a été testée par Thille et al. [6] dans une seconde étude autopsique utilisant la présence de DAD histologique comme gold standard. Tous les patients qui décédaient en réanimation dans un contexte de SDRA étaient classés selon les trois catégories de la définition de Berlin. La quasi-totalité des patients ayant des lésions de DAD présentaient les critères de Berlin [2]. À l'inverse, moins de la moitié de ceux présentant un SDRA radioclinique avaient 
Tableau 1 Définitions du SDRA : comparaison des définitions de l'AECC (1994) [11] et de Berlin (2012) [2]

\begin{tabular}{|c|c|c|c|}
\hline & Définition AECC & Limites AECC & Définition de Berlin \\
\hline Délai d'installation & Apparition aiguë & Terme aigu non défini & $\begin{array}{l}\text { Dans les } 7 \text { jours suivant l'agression } \\
\text { ou l'aggravation des symptômes } \\
\text { respiratoires }\end{array}$ \\
\hline Oxygénation & $\begin{array}{l}\text { Deux entités : } \\
\text { ALI : } \mathrm{PaO}_{2} / \mathrm{FiO}_{2} \leq 300 \mathrm{mmHg} \\
\text { SDRA : } \mathrm{PaO}_{2} / \mathrm{FiO}_{2} \leq 200 \mathrm{mmHg}\end{array}$ & $\begin{array}{l}\text { ALI souvent interprété comme } \\
\mathrm{PaO}_{2} / \mathrm{FiO}_{2}=201-300 \mathrm{mmHg} \\
\text { Confusion entre ALI/SDRA }\end{array}$ & $\begin{array}{l}\text { Séparation en } 3 \text { groupes : } \\
\text { Léger : } 200 \mathrm{mmHg}<\mathrm{PaO}_{2} / \mathrm{FiO}_{2} \leq \\
300 \mathrm{mmHg} \\
\text { Modéré : } 100 \mathrm{mmHg}<\mathrm{PaO}_{2} / \mathrm{FiO}_{2} \leq \\
200 \mathrm{mmHg} \\
\text { Sévère : } \mathrm{PaO}_{2} / \mathrm{FiO}_{2} \leq 100 \mathrm{mmHg}\end{array}$ \\
\hline Condition ventilatoire & Non définie & $\begin{array}{l}\text { Problème de fluctuation } \\
\text { du rapport } \mathrm{PaO}_{2} / \mathrm{FiO}_{2} \\
\text { selon le niveau de PEP et la } \mathrm{FiO}_{2} \\
\text { Patients en VNI exclus } \\
\text { de la définition du SDRA }\end{array}$ & $\begin{array}{l}\text { Le rapport } \mathrm{PaO}_{2} / \mathrm{FiO}_{2} \text { doit être } \\
\text { mesuré avec une } \mathrm{PEP} \geq 5 \mathrm{cmH} 2 \mathrm{O} \\
\text { Les patients en pression positive } \\
\text { continue (par extension, } \\
\text { oxygénothérapie à haut débit } \\
\text { et VNI) sont classés dans les SDRA } \\
\text { légers }\end{array}$ \\
\hline $\begin{array}{l}\text { Radiographie } \\
\text { thoracique }\end{array}$ & $\begin{array}{l}\text { Infiltrats bilatéraux } \\
\text { sur la radiographie thoracique } \\
\text { de face }\end{array}$ & $\begin{array}{l}\text { Type d'infiltrats non défini } \\
\text { Variabilité de l'interprétation } \\
\text { de la radiographie thoracique }\end{array}$ & $\begin{array}{l}\text { Opacités bilatérales non expliquées } \\
\text { complètement par un épanchement, } \\
\text { un collapsus pulmonaire } \\
\text { ou des nodules }\end{array}$ \\
\hline PAPO & $\begin{array}{l}\text { PAPO } \leq 18 \mathrm{mmHg} \text { si mesurée } \\
\text { ou absence d'arguments } \\
\text { cliniques pour une hyperpression } \\
\text { de l'oreillette gauche }\end{array}$ & $\begin{array}{l}\text { Seuil de } 18 \mathrm{mmHg} \text { arbitraire } \\
\text { PAPO élevée et SDRA peuvent } \\
\text { coexister } \\
\text { Variabilité de mesure } \\
\text { et d'interprétation de la PAPO } \\
\text { entre les cliniciens }\end{array}$ & $\begin{array}{l}\text { Tableau respiratoire non } \\
\text { complètement expliqué } \\
\text { par une insuffisance cardiaque } \\
\text { ou une surcharge hydrosodée } \\
\text { Critère PAPO absent de la définition }\end{array}$ \\
\hline $\begin{array}{l}\text { Exposition } \\
\text { à un facteur de risque }\end{array}$ & Non définie & Non incluse dans la définition & $\begin{array}{l}\text { Incluse dans la définition } \\
\text { Nécessité d'évaluer objectivement } \\
\text { les pressions de remplissage du cœur } \\
\text { gauche en l'absence de facteurs } \\
\text { de risque identifiés }\end{array}$ \\
\hline
\end{tabular}

des lésions de DAD à l'examen histologique. Ainsi, la sensibilité ( $89 \%$ ) était meilleure que celle de la définition précédente, mais la spécificité était moins bonne (63\%). Au maximum, $70 \%$ des patients SDRA, selon la définition de Berlin [2], présentaient un DAD histologique, et ce, dans le sous-groupe des patients remplissant les critères de SDRA sévère pendant plus de 72 heures [6] (Fig. 2). Il convient de ne pas perdre de vue l'existence, dans ces études autopsiques, d'un biais de sélection majeur qui surestime potentiellement la prévalence du DAD en sélectionnant les patients les plus sévères (c'est-à-dire ceux qui sont décédés). En fait, la prévalence réelle du DAD chez les patients remplissant les critères radiocliniques de SDRA n'est pas connue, et les données histologiques issues des séries autopsiques discutées ci-dessus [6,7] — tout comme celles issues de cohortes de patients ayant subi une biopsie pulmonaire chirurgicale [13-15] — remettent en cause le DAD comme seul substrat histologique du SDRA. Une hypothèse alternative est que le DAD ne soit qu'un témoin de la sévérité du SDRA, voire un marqueur de lésions pulmonaires induites par la ventilation mécanique. Cela est suggéré dans l'étude de Thille et al. [6] par la moindre prévalence du DAD chez les patients décédés de SDRA entre 2001 et 2010, ventilés avec un volume courant réduit, comparativement à ceux décédés entre 1991 et 2000 , ventilés avec un volume courant plus élevé. Compte tenu de l'absence de données histologiques chez la plupart 


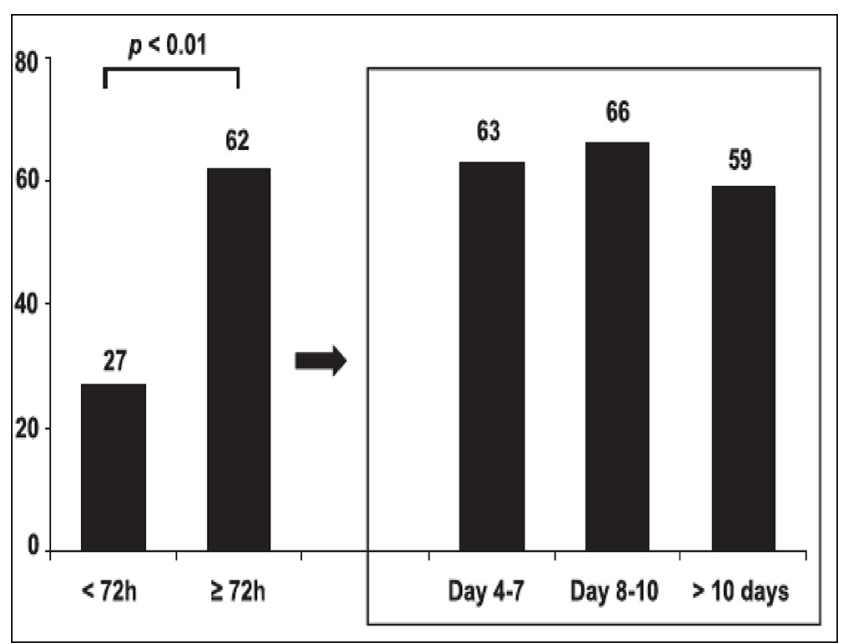

Fig. 2 Proportion de patients atteints et décédés de SDRA modéré ou sévère (définition de Berlin [2]) chez lesquels des lésions de dommage alvéolaire diffus (DAD) étaient mises en évidence à l'autopsie, en fonction de la durée depuis laquelle les critères de SDRA étaient présents. La proportion de DAD était significativement plus élevée chez les patients qui avaient les critères de SDRA depuis plus de 72 heures. Parmi ces patients, la proportion de DAD restait stable quel que soit le délai entre la présence des critères de SDRA et le décès (d'après Thille et al. [6], avec autorisation)

des patients ayant un SDRA, et dans la mesure où la présence de DAD pourrait être un marqueur de lésions pulmonaires induites par la ventilation mécanique, un certain nombre d'experts s'accordent aujourd'hui à considérer que les SDRA radiocliniques (tels que définis par le consensus de Berlin [2]) recoupent une diversité d'histologies sousjacentes.

Par conséquent, il existe un sous-groupe de patients n'ayant pas de DAD histologique, mais remplissant les critères radiocliniques de Berlin [2]. Ainsi, dans l'étude de Thille et al. [6], parmi les patients qui n'avaient pas de DAD à l'autopsie, une variété de diagnostics histologiques alternatifs étaient retrouvés : pneumonie (49\%), abcès pulmonaire $(3 \%)$, œdème aigu du poumon $(4,5 \%)$, infiltration néoplasique du poumon $(4,5 \%)$, hémorragie intra-alvéolaire (6\%), pneumopathie interstitielle ou fibrose pulmonaire $(4,5 \%)$, emphysème $(7 \%)$ et absence de lésions histologiques dans $14 \%$ des cas [6]. Les séries histologiques issues de patients SDRA ayant eu une biopsie pulmonaire chirurgicale retrouvent une prévalence des SDRA sans DAD de l'ordre de $50 \%$ et rapportent une variété d'entités histologiques (Tableau 2) : infiltrations néoplasiques du poumon, pneumopathies infiltrantes diffuses d'origine immune, médicamenteuse ou idiopathique $[15,18,33,34]$. De manière intéressante, le phénotype clinique des patients ayant un SDRA avec DAD semble différer de celui des patients sans DAD : âge plus élevé, oxygénation et mécanique respiratoire plus altérées ainsi qu'une probabilité plus élevée de décéder d'hypoxémie réfractaire [14]. A contrario, il n'existe pas à ce jour de données suggérant que l'histologie du SDRA pourrait avoir un impact sur la réponse à une intervention thérapeutique, telle que la mise en décubitus ventral $[13,35]$, ou sur la survie des patients. Cependant, la prévalence des SDRA sans DAD et la variété des étiologies des SDRA sans facteurs de risque « communs » suggèrent qu'une prise en charge personnalisée de ces patients pourrait être bénéfique.

\section{Facteurs de risque de SDRA et SDRA sans facteurs de risque retrouvés}

Parmi les facteurs de risque connus de SDRA, la plupart ont été listés dans la définition de l'AECC [11], puis repris dans la définition de Berlin [2] sous le terme de facteurs de risque « communs ». Ces facteurs de risque ont été historiquement classés en deux catégories : pulmonaire ou directe et extrapulmonaire ou indirecte (Tableau 3). Les facteurs de risque les plus fréquemment rencontrés, d'après des données issues de la cohorte Lung Safe [1], sont : la pneumonie infectieuse, l'inhalation de liquide gastrique et le sepsis. Tous les facteurs de risque n'entraînent pas le même risque de développer un SDRA [36], et le risque de développer un SDRA augmente avec le nombre de facteurs de risque présents chez un même patient. La ventilation mécanique en pression positive n'apparaît pas en tant que telle parmi les facteurs de risque de Berlin, mais une littérature expérimentale et clinique abondante a bien souligné son rôle central, au moins dans l'aggravation des lésions pulmonaires ayant conduit à son initiation $[3,37]$.

Comme discuté plus haut, une fraction importante des patients atteints de SDRA ont, à l'examen histologique du poumon, d'autres lésions que le DAD [14,34]. Identifier ces patients est potentiellement important dans la mesure où certaines lésions histologiques sont considérées comme corticosensibles. Diagnostiquer ces anomalies histologiques « non$\mathrm{DAD} »$ pourrait ainsi conduire à une prise en charge thérapeutique ciblée de ces patients. Or, le recours à la biopsie pulmonaire chirurgicale reste marginal au cours du SDRA [19]. Ainsi, certains experts ont suggéré d'isoler un sousgroupe particulier de patients SDRA chez lesquels la probabilité d'avoir un diagnostic atypique est plus élevée : il s'agit des patients chez lesquels aucun facteur de risque de SDRA n'a été décelé au moment du diagnostic du SDRA [33]. Deux études ont étudié la prévalence et les étiologies de ces SDRA sans facteurs de risque retrouvés :

- Dans une étude rétrospective bicentrique, ayant inclus 702 patients atteints de SDRA, la prévalence du SDRA sans facteurs de risque était de 7,5\% [16]. Une cause au SDRA était retrouvée dans plus de $75 \%$ des cas 


\begin{tabular}{|c|c|c|c|c|}
\hline Cadre diagnostique & $\begin{array}{l}\text { Principales causes/cadre } \\
\text { diagnostique }\end{array}$ & $\begin{array}{l}\text { Caractéristiques } \\
\text { radiographiques }\end{array}$ & $\begin{array}{l}\text { Lavage } \\
\text { bronchoalvéolaire/ } \\
\text { histologie }\end{array}$ & $\begin{array}{l}\text { Références } \\
\text { pratiques }\end{array}$ \\
\hline SDRA médicamenteux & $\begin{array}{l}\text { Amiodarone, méthotrexate, } \beta \text { - } \\
\text { bloquants, bléomycine, } \\
\text { cyclophosphamide, rituximab, } \\
\text { médicaments anticancéreux }\end{array}$ & $\begin{array}{l}\text { Opacités interstitielles } \\
\text { ou alvéolaires }\end{array}$ & $\begin{array}{l}\text { Alvéolite lymphocytaire, } \\
\text { hémorragie intra- } \\
\text { alvéolaire }\end{array}$ & $\begin{array}{l}{[16,17]} \\
\text { Pneumo- } \\
\text { tox }^{\circledR}\end{array}$ \\
\hline $\begin{array}{l}\text { SDRA d'origine } \\
\text { immune }\end{array}$ & $\begin{array}{l}\text { Vascularites des petits vaisseaux, } \\
\text { connectivités }\end{array}$ & $\begin{array}{l}\text { Verre dépoli, opacités } \\
\text { alvéolaires confluentes, } \\
\text { nodules excavés }\end{array}$ & $\begin{array}{l}\text { Hypercellulaire } \\
\text { Hémorragie intra- } \\
\text { alvéolaire fréquente }\end{array}$ & {$[16,18]$} \\
\hline SDRA néoplasique & $\begin{array}{l}\text { Lymphangite carcinomateuse, } \\
\text { hémopathies malignes }\end{array}$ & $\begin{array}{l}\text { Nodules/masses, } \\
\text { adénopathies } \\
\text { médiastinales, } \\
\text { épanchement pleural }\end{array}$ & $\begin{array}{l}\text { Alvéolite lymphocytaire, } \\
\text { cellules tumorales }\end{array}$ & {$[16,19]$} \\
\hline \multirow[t]{6}{*}{ Causes rares de SDRA } & $\begin{array}{l}\text { Exacerbation de fibrose } \\
\text { pulmonaire idiopathique, } \\
\text { aggravation de pneumonie } \\
\text { infiltrante diffuse }\end{array}$ & $\begin{array}{l}\text { Nouvelles opacités } \\
\text { en verre dépoli } \\
\text { ou consolidations } \\
\text { sur un fond de lésions } \\
\text { de type PIC (rayon de miel, } \\
\text { bronchiectasies...) }\end{array}$ & Alvéolite neutrophilique & {$[20,21]$} \\
\hline & $\begin{array}{l}\text { Pneumonie aiguë idiopathique } \\
\text { (ex. syndrome d'Hamman-Rich) }\end{array}$ & $\begin{array}{l}\text { Condensations alvéolaires } \\
\text { bilatérales, verre dépoli, } \\
\text { rayon de miel }\end{array}$ & $\begin{array}{l}\text { Alvéolite neutrophilique } \\
\text { Dommage alvéolaire } \\
\text { diffus }\end{array}$ & {$[22,23]$} \\
\hline & $\begin{array}{l}\text { Pneumonie organisée } \\
\text { cryptogénique ou secondaire } \\
\text { (postinfectieuse, postradique, } \\
\text { médicamenteuse, etc.) }\end{array}$ & $\begin{array}{l}\text { Condensations alvéolaires } \\
\text { sous-pleurales, parfois } \\
\text { migratrices, verre dépoli } \\
\text { patchy, signe du halo } \\
\text { inversé (rarement) }\end{array}$ & $\begin{array}{l}\text { Hypercellulaire, panaché } \\
(\mathrm{PNN}, \text { lymphocytes }< \\
25 \% ; \text { PNE }<25 \%)\end{array}$ & {$[24,25]$} \\
\hline & $\begin{array}{l}\text { Hémorragie intra-alvéolaire } \\
\text { diffuse (immunes, } \\
\text { médicamenteuses, infectieuses, } \\
\text { néoplasiques) }\end{array}$ & $\begin{array}{l}\text { Non spécifique, verre } \\
\text { dépoli hétérogène fréquent }\end{array}$ & $\begin{array}{l}\text { Macroscopiquement } \\
\text { hémorragique ; signe } \\
\text { des trois seringues } \\
\text { (aspect de plus en plus } \\
\text { rouge du LBA) }\end{array}$ & {$[26-28]$} \\
\hline & $\begin{array}{l}\text { Pneumopathies d'hypersensibilité } \\
\text { (allergènes spécifiques « poumons } \\
\text { de fermier » ou « éleveurs } \\
\text { d'oiseaux », médicaments) }\end{array}$ & Verre dépoli, micronodules & $\begin{array}{l}\text { Alvéolite lymphocytaire } \\
\text { (rapport CD4/CD8 < 1) }\end{array}$ & {$[29,30]$} \\
\hline & $\begin{array}{l}\text { Pneumopathie } \\
\text { hyperéosinophilique } \\
\text { (médicamenteuse, immune, } \\
\text { associée à un clone T, } \\
\text { à un syndrome myéloprolifératif, } \\
\text { ou idiopathique) }\end{array}$ & $\begin{array}{l}\text { Opacités interstitielles } \\
\text { ou alvéolaires diffuses, } \\
\text { aspect en } O A P \text { inversé } \\
\text { épanchement pleural }\end{array}$ & $\begin{array}{l}\text { Alvéolite éosinophile } \\
(\mathrm{PNE}>25 \%)\end{array}$ & {$[31,32]$} \\
\hline
\end{tabular}

LBA : lavage bronchoalvéolaire ; PNN : polynucléaires neutrophiles ; PNE : polynucléaires éosinophiles ; PIC : pneumopathie interstitielle commune 
Tableau 3 Facteurs de risque « communs » de SDRA selon la définition de Berlin [2]

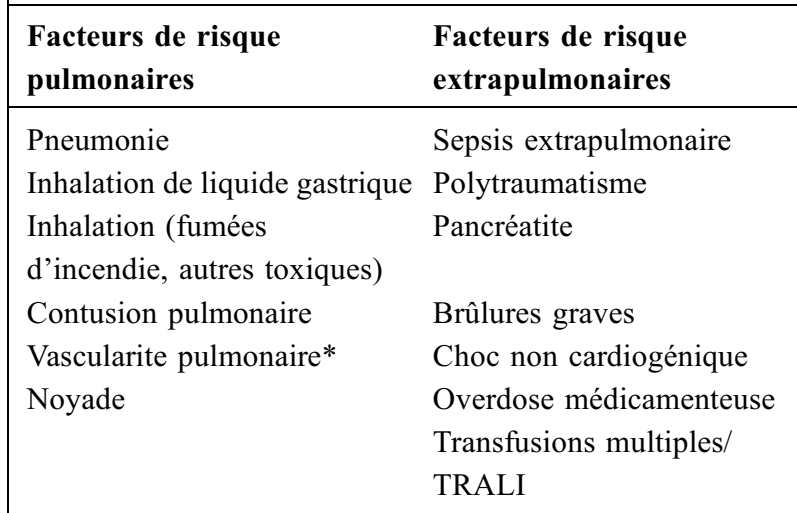

TRALI : transfusion-related acute lung injury

* Les vascularites pulmonaires appartiennent à la liste des facteurs de risque « communs » de SDRA dans la définition de Berlin [2], mais, en pratique, celles-ci appartiennent aux causes de SDRA d'origine immune, car elles relèvent d'un traitement immunosuppresseur (Tableau 2).

(principalement d'origine immune, médicamenteuse ou néoplasique) et, de manière intéressante, l'absence de facteur de risque de SDRA était un facteur de risque de mortalité en réanimation, y compris après ajustement, notamment sur les scores de sévérité à l'admission et sur le rapport $\mathrm{PaO}_{2} / \mathrm{FiO}_{2}[16]$;

- Une seconde étude (l'étude Asteroid), prospective internationale, ancillaire de l'étude Lung Safe [1], a retrouvé chez 2813 patients admis pour SDRA une prévalence des SDRA sans facteurs de risque de $8,3 \%$, donc comparable à celle de l'étude précédente [19]. Cependant, cette étude ne retrouvait pas d'impact négatif de l'absence de facteur de risque sur la mortalité des patients, contrairement à l'étude précitée [16]. Par ailleurs, cette étude rapportait que, chez près de $80 \%$ de ces patients SDRA sans facteurs de risque " communs » décelés, aucune étiologie n'était retrouvée. Ces données soulignent la nécessité d'une approche diagnostique standardisée, notamment dans les situations où aucun facteur de risque n'est identifié après une première évaluation.

\section{Explorations diagnostiques}

Les améliorations récentes du pronostic du SDRA sont essentiellement dues à une meilleure prise en charge symptomatique (ventilation protectrice, décubitus ventral précoce, gestion restrictive de la balance hydrique et de la sédation, prévention des pneumonies acquises sous ventilation mécanique). Cependant, il paraît essentiel de ne pas négliger l'enquête diagnostique et d'identifier dès que possible la cause de l'agression pulmonaire à l'origine du tableau de SDRA, afin d'adapter le traitement étiologique. Il est ainsi frappant de noter que dans l'étude Asteroid [19], parmi les $8 \%$ des patients SDRA qui n'avaient pas de facteurs de risque objectivés, la cause du SDRA n'avait pas été élucidée pour $80 \%$ d'entre eux à la fin de leur hospitalisation en réanimation [19]. L'enjeu est de ne pas retarder l'initiation d'un traitement spécifique (arrêt d'un médicament imputable à une pneumonie médicamenteuse, immunosuppression pour les causes immunes, chimiothérapie pour les SDRA d'origine néoplasique, etc.). L'étude observationnelle de Gibelin et al. [16] suggérait un bénéfice des corticoïdes dans le sous-groupe des SDRA sans facteurs de risque identifiés, allant dans le sens des données de la littérature qui plaident pour une immunosuppression précoce chez ces patients $[16,38]$. Le niveau de preuve du recours à la corticothérapie chez ces patients est néanmoins très faible, et il apparaît indispensable d'évaluer le rapport bénéfice/risque d'une telle intervention pharmacologique au cas par cas et donc de poser un diagnostic étiologique précis.

L'enquête étiologique s'efforcera dans un premier temps de rechercher les facteurs de risque communs de SDRA (Fig. 3) [2]. En raison de la fréquence des infections, notamment respiratoires, parmi les causes de SDRA, l'enquête microbiologique doit être large et systématique : hémocultures, antigénuries légionnelle et pneumocoque, prélèvement endobronchique, si le patient est ventilé, ou examen cytobactériologique des crachats dans le cas contraire. Les sérologies des germes intracellulaires sont utiles lorsqu'elles sont positives, mais ne permettent d'éliminer une infection à germe atypique avec une bonne sensibilité qu'après réalisation d'une sérologie de contrôle à distance. Le lavage bronchoalvéolaire (LBA) est un examen de choix, car il permet de rechercher un large panel de pathogènes (bactéries, virus, champignons et parasites) [39]. L'identification des virus respiratoires, responsables d'un tiers des pneumonies sévères [40], est actuellement facilitée par l'utilisation des techniques de polymerase chain reaction (PCR) multiplex avec des panels ciblant les virus respiratoires et les bactéries intracellulaires (c'est-à-dire Mycoplasma pneumoniae, Chlamydia pneumoniae, Legionella pneumophila et Bordetella pertussis). Ces PCR peuvent être réalisées sur un prélèvement nasopharyngé ou directement sur le liquide du LBA. La recherche d'une immunosuppression est évidemment fondamentale (sérologie VIH) à ce stade.

Lorsqu'aucun facteur de risque infectieux n'est mis en évidence, et avant d'aborder des explorations plus poussées, il est indispensable d'éliminer une origine cardiogénique au tableau respiratoire aigu par une évaluation objective des pressions de remplissage du ventricule gauche, comme préconisé par la définition de Berlin [2]. Dans l'étude Asteroid [19], chez plus de $30 \%$ des patients ayant un SDRA sans 
facteurs de risque identifiés, aucune évaluation des pressions de remplissage du cœur gauche n'avait été réalisée. Si l'origine cardiogénique est écartée, des explorations plus spécifiques deviennent nécessaires afin de rechercher une cause possiblement réversible du SDRA, incluant un bilan immunologique large (comprenant, mais non limité à, facteurs antinucléaires, anticorps anti-ADN natifs, anti-antigènes nucléaires solubles, ANCA, anticorps antisynthétases et autres anticorps associés aux myosites, facteur rhumatoïde, anticorps anti-CCP, électrophorèse des protides), la recherche des pneumopathies d'hypersensibilité (sérologies « éleveur d'oiseaux » et " poumon de fermier ») et une enquête médicamenteuse (se référer au site pneumotox.com ou à l'application Pneumotox disponible pour smartphone). Cette énumération n'est évidemment pas exhaustive et sera modulée au cas par cas, après discussion avec d'autres spécialistes (pneumologue, interniste, rhumatologue, etc.), en fonction du terrain et de l'histoire clinique du patient.

La réalisation d'un scanner thoracique en coupes fines est souvent rentable à ce stade. La mise en évidence de certains « patterns » tomodensitométriques pourrait avoir un intérêt diagnostique (par exemple adénopathies médiastinales, masses orientant vers une néoplasie) ou pronostique. Ainsi, la prédominance de verre dépoli est évocatrice d'une cause réversible, tandis que la prédominance de lésions kystiques en rayon de miel suggère une fibrose pulmonaire déjà avancée et est généralement associée à un mauvais pronostic $[16,33]$.

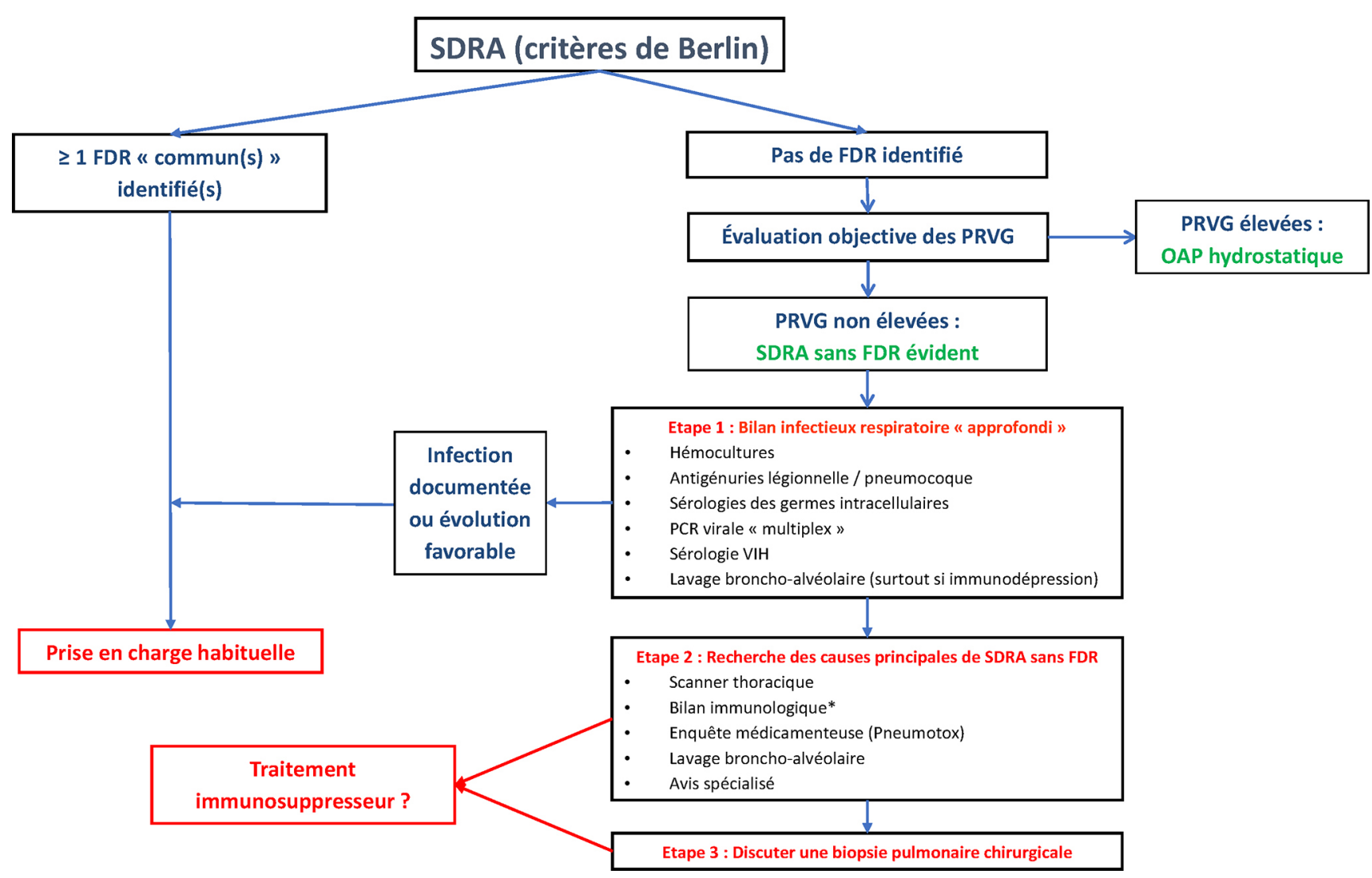

Fig. 3 Stratégie diagnostique pour les patients atteints de SDRA. La première étape est de rechercher la présence d'un ou de plusieurs facteurs de risque (FDR) « communs » de SDRA [2]. En leur absence, il faut impérativement s'assurer de l'absence d'élévation des pressions de remplissage du cœur gauche (PRVG) en utilisant une méthode objective (par exemple, échocardiographie ou autre). Si les PRVG ne sont pas élevées, nous proposons de réaliser un bilan infectieux approfondi, puis, en cas de négativité, de rechercher les causes principales du SDRA sans FDR (Tableau 2). En fonction du cadre diagnostique, une biopsie pulmonaire chirurgicale pourra être envisagée, et un traitement immunosuppresseur (corticoïdes le plus souvent) sera discuté. Le lavage bronchoalvéolaire sera envoyé au laboratoire pour bactériologie (incluant bactériologie standard, recherche de mycobactéries et de germes à croissance lente), virologie et cytologie pour les sujets immunocompétents ; pour les sujets immunodéprimés, une analyse mycoparasitologique sera nécessaire. $*$ comprenant notamment : facteurs antinucléaires, anticorps anti-ADN natifs, anti-antigènes nucléaires solubles, ANCA, facteur rhumatoïde, anticorps anti-CCP, anticorps antisynthétases et autres anticorps associés aux myosites, sérologies « éleveur d'oiseaux » et « poumon de fermier », enzyme de conversion de l'angiotensine (liste non exhaustive) 
Malgré des explorations exhaustives, le diagnostic étiologique reste parfois énigmatique, et le recours à la biopsie pulmonaire chirurgicale peut se discuter en cas de SDRA « persistant ", c'est-à-dire dont l'évolution clinique est défavorable après sept jours [33,41]. Il existe deux bénéfices théoriques à la réalisation d'une biopsie pulmonaire chirurgicale :

- faire le diagnostic précoce d'une étiologie curable, notamment infectieuse, lorsque les explorations réalisées sont négatives ;

- documenter une fibroprolifération qui motiverait l'initiation d'une corticothérapie, même si des données récentes plaident pour l'utilisation d'une approche moins invasive, telle que le dosage du procollagène 3 dans le LBA, qui est bien corrélé à la présence de fibrose histologique [42].

En pratique, le recours à la biopsie pulmonaire chirurgicale est aujourd'hui anecdotique ; ainsi, dans l'étude Asteroid [19], seuls 2,6 \% des patients sans facteurs de risque retrouvé avaient une biopsie pulmonaire chirurgicale, tandis que ce taux diminuait à $0,2 \%$ dans le groupe des SDRA avec facteurs de risque. En définitive, une biopsie pulmonaire chirurgicale ne sera envisagée que lorsqu'un bilan étiologique complet a été réalisé et n'est pas contributif, ou lorsqu'un résultat contributif à la biopsie est fortement suspecté et est susceptible d'induire une modification thérapeutique $[34,43]$. Dans tous les cas, la biopsie sera réalisée par une équipe médicale et chirurgicale expérimentée, afin de limiter les complications liées à la procédure [41].

\section{Pronostic du SDRA}

Le pronostic du SDRA a été transformé au cours des dernières décennies grâce à l'amélioration des connaissances sur la physiopathologie des lésions pulmonaires induites par la ventilation mécanique ayant permis la réalisation d'essais cliniques qui ont conduit à une modification des pratiques de prise en charge des patients ayant un SDRA. Ainsi, la diminution du volume courant [4], la mise en décubitus ventral [44] et la curarisation [45] précoce chez les patients ayant un rapport $\mathrm{PaO}_{2} / \mathrm{FiO}_{2}$ inférieur à $150 \mathrm{mmHg}$ ont permis une réduction importante de la mortalité du SDRA. Le SDRA représente, cependant, toujours un problème de santé publique important, avec une mortalité hospitalière - toutes causes confondues - autour de $40 \%$, selon les données de l'étude Lung Safe. Cette étude a, en outre, mis en évidence une application encore trop limitée de la réduction du volume courant et du décubitus ventral (16\% seulement dans le sous-groupe des SDRA sévères) [1]. Cette étude a également pointé le problème du sous-diagnostic de SDRA par les cliniciens, associé à une altération de la prise en charge des patients en termes de recours à la ventilation pro- tectrice ou au décubitus ventral. De manière intéressante, l'absence de facteur de risque de SDRA était, en tant que telle, aussi associée à un sous-diagnostic du SDRA, suggérant là encore l'importance d'adopter une démarche diagnostique systématique.

L'évaluation du pronostic des patients ayant un SDRA sans facteurs de risque identifié dans l'étude Asteroid [19] n'a pas mis en évidence de différence significative en termes de mortalité hospitalière par rapport aux patients ayant un facteur de risque de SDRA, et ce, même après appariement de ces deux groupes de patients selon un score de propension. Ce score permettait d'ajuster un nombre important de facteurs associés à la présence ou non d'un facteur de risque de SDRA. L'analyse de ces résultats doit cependant tenir compte, comme discuté plus haut, du fait que $30 \%$ des patients sans facteurs de risque identifié n'avaient pas eu d'évaluation objective des pressions de remplissage du cœur gauche [19], suggérant que certains d'entre eux avaient peutêtre d'authentiques œdèmes aigus cardiogéniques du poumon. Par ailleurs, les investigations diagnostiques (LBA, scanner thoracique, bilan d'auto-immunité...) n'étaient pas conduites de façon exhaustive [19]. Il en résultait que la cause du SDRA n'était pas identifiée chez $80 \%$ des patients sans facteurs de risque et que le recours à la corticothérapie dans ce sous-groupe était plus faible qu'attendu (17,5\%) et étonnamment comparable à celui des SDRA avec facteur de risque $(18,1 \%)$ [19]. La standardisation du bilan diagnostique apparaît donc indispensable, afin de réduire la fraction de patients ayant un SDRA sans cause clairement identifiée pour permettre une prise en charge spécifique en fonction du diagnostic retenu. La validation d'algorithmes guidant les explorations diagnostiques chez les patients ayant un SDRA dans le cadre d'études prospectives semble donc nécessaire.

\section{Conclusion}

Le SDRA est une entité clinicoradiologique hétérogène qui a été historiquement rattachée à la présence de DAD histologique. Cependant, une proportion non négligeable de patients ayant un SDRA ont une autre histologie, et la présence de DAD pourrait être un marqueur de lésions pulmonaires induites par la ventilation mécanique. L'identification rapide du ou des facteurs de risque de SDRA est un élément clé de la prise en charge initiale. Une stratégie diagnostique standardisée doit être mise en œuvre sans délai, lorsqu'aucun facteur de risque n'est décelé, afin de permettre une prise en charge adaptée à la cause du SDRA.

Liens d'intérêts : les auteurs déclarent ne pas avoir de lien d'intérêt. 


\section{Références}

1. Bellani G, Laffey JG, Pham T, Fan E, Brochard L, Esteban A, Gattinoni L, van Haren F, Larsson A, McAuley DF, Ranieri M, Rubenfeld G, Thompson BT, Wrigge H, Slutsky AS, Pesenti A, (2016) Epidemiology, patterns of care, and mortality for patients with acute respiratory distress syndrome in intensive care units in 50 countries. JAMA 315: 788-800

2. Ranieri VM, Rubenfeld GD, Thompson BT, Ferguson ND, Caldwell E, Fan E, Camporota L, Slutsky AS, (2012) Acute respiratory distress syndrome: the Berlin definition. JAMA 307: 2526-33

3. Dreyfuss D, Saumon G, (1998) Ventilator-induced lung injury. Am J Respir Crit Care Med 157: 294-323

4. Brower RG, Matthay MA, Morris A, Schoenfeld D, Thompson BT, Wheeler A; Acute Respiratory Distress Syndrome Network (2000) Ventilation with lower tidal volumes as compared with traditional tidal volumes for acute lung injury and the acute respiratory distress syndrome. N Engl J Med 342: 1301-1308

5. Katzenstein AL, Bloor CM, Leibow AA, (1976) Diffuse alveolar damage--the role of oxygen, shock, and related factors. A review. Am J Pathol 85: 209-228

6. Thille AW, Esteban A, Fernández-Segoviano P, Rodriguez JM, Aramburu JA, Peñuelas O, Cortés-Puch I, Cardinal-Fernández P, Lorente JA, Frutos-Vivar F, (2013) Comparison of the Berlin definition for acute respiratory distress syndrome with autopsy. Am J Respir Crit Care Med 187: 761-767

7. Esteban A, Fernández-Segoviano P, Frutos-Vivar F, Aramburu JA, Nájera L, Ferguson ND, Alía I, Gordo F, Ríos F, (2004) Comparison of clinical criteria for the acute respiratory distress syndrome with autopsy findings. Ann Intern Med 141: 440-445

8. Ashbaugh DG, Bigelow DB, Petty T, Levine BE, (1967) Acute respiratory distress in adults. Lancet 2: 319-323

9. Tomashefski JF, (2000) Pulmonary pathology of acute respiratory distress syndrome. Clin Chest Med 21: 435-466

10. Thille AW, Esteban A, Fernández-Segoviano P, Rodriguez JM, Aramburu JA, Vargas-Errázuriz P, Martín-Pellicer A, Lorente JA, Frutos-Vivar F, (2013) Chronology of histological lesions in acute respiratory distress syndrome with diffuse alveolar damage: a prospective cohort study of clinical autopsies. Lancet Respir Med 1: 395-401

11. Bernard GR, Artigas A, Brigham KL, Carlet J, Falke K, Hudson L, Lamy M, Legall JR, Morris A, Spragg R, (1994) The American-European Consensus Conference on ARDS. Definitions, mechanisms, relevant outcomes, and clinical trial coordination. Am J Respir Crit Care Med 149: 818-824

12. Ferguson ND, Fan E, Camporota L, Antonelli M, Anzueto A, Beale R, Brochard L, Brower R, Esteban A, Gattinoni L, Rhodes A, Slutsky AS, Vincent JL, Rubenfeld GD, Thompson BT, Ranieri VM, (2012) The Berlin definition of ARDS: an expanded rationale, justification, and supplementary material. Intensive Care Med 38: 1573-1582

13. Guerin C, Bayle F, Leray V, Debord S, Stoian A, Yonis H, Roudaut JB, Bourdin G, Devouassoux-Shisheboran M, Bucher E, Ayzac L, Lantuejoul S, Philipponnet C, Kemeny JL, Souweine B, Richard JC, (2015) Open lung biopsy in nonresolving ARDS frequently identifies diffuse alveolar damage regardless of the severity stage and may have implications for patient management. Intensive Care Med 41: 222-230

14. Lorente JA, Cardinal-Fernández P, Muñoz D, Frutos-Vivar F, Thille AW, Jaramillo C, Ballén-Barragán A, Rodríguez JM, Peñuelas O, Ortiz G, Blanco J, Pinheiro BV, Nin N, del Carmen Marin M, Esteban A, Thompson TB, (2015) Acute respiratory distress syndrome in patients with and without diffuse alveolar damage: an autopsy study. Intensive Care Med 41: 1921-1930
15. Kao KC, Hu HC, Chang CH, Hung CY, Chiu LC, Li SH, Lin SW, Chuang LP, Wang CW, Li LF, Chen NH, Yang CT, Huang CC, Tsai YH, (2015) Diffuse alveolar damage associated mortality in selected acute respiratory distress syndrome patients with open lung biopsy. Crit Care 19: 228

16. Gibelin A, Parrot A, Maitre B, Brun-Buisson C, Mekontso Dessap A, Fartoukh M, de Prost N, (2016) Acute respiratory distress syndrome mimickers lacking common risk factors of the Berlin definition. Intensive Care Med 42: 164-172

17. Dhokarh R, Li G, Schmickl CN, Kashyap R, Assudani J, Limper AH, Gajic O, (2012) Drug-associated acute lung injury: a population-based cohort study. Chest 142: 845-850

18. Patel SR, Karmpaliotis D, Ayas NT, Mark EJ, Wain J, Thompson BT, Malhotra A, (2004) The role of open-lung biopsy in ARDS. Chest 125: 197-202

19. de Prost N, Pham T, Carteaux G, Mekontso Dessap A, BrunBuisson C, Fan E, Bellani G, Laffey J, Mercat A, Brochard L, Maître B ; LUNG SAFE investigators; ESICM trials group; REVA network, (2017) Etiologies, diagnostic work-up and outcomes of acute respiratory distress syndrome with no common risk factor: a prospective multicenter study. Ann Intensive Care 7: 69

20. Zafrani L, Lemiale V, Lapidus N, Lorillon G, Schlemmer B, Azoulay E, (2014) Acute respiratory failure in critically ill patients with interstitial lung disease. PLoS One 9: e104897

21. Collard HR, Ryerson CJ, Corte TJ, Jenkins G, Kondoh Y, Lederer DJ, Lee JS, Maher TM, Wells AU, Antoniou KM, Behr J, Brown KK, Cottin V, Flaherty KR, Fukuoka J, Hansell DM, Johkoh T, Kaminski N, Kim DS, Kolb M, Lynch DA, Myers JL, Raghu G, Richeldi L, Taniguchi H, Martinez FJ, (2016) Acute exacerbation of idiopathic pulmonary fibrosis. An international working group report. Am J Respir Crit Care Med 194: 265-275

22. Vourlekis JS, Brown KK, Cool CD, Young DA, Cherniack RM, King TE, Schwarz MI, (2000) Acute interstitial pneumonitis. Case series and review of the literature. Medicine (Baltimore) 79: $369-378$

23. Suh GY, Kang EH, Chung MP, Lee KS, Han J, Kitaichi M, Kwon OJ, (2006) Early intervention can improve clinical outcome of acute interstitial pneumonia. Chest 129: 753-761

24. Drakopanagiotakis F, Paschalaki K, Abu-Hijleh M, Aswad B, Karagianidis N, Kastanakis E, Braman SS, Polychronopoulos V, (2011) Cryptogenic and secondary organizing pneumonia: clinical presentation, radiographic findings, treatment response, and prognosis. Chest 139: 893-900

25. Baque-Juston M, Pellegrin A, Leroy S, Marquette $\mathrm{CH}$, Padovani B, (2014) Organizing pneumonia: what is it? A conceptual approach and pictorial review. Diagn Interv Imaging 95: 771-777

26. Leatherman JW, Davies SF, Hoidal JR, (1984) Alveolar hemorrhage syndromes: diffuse microvascular lung hemorrhage in immune and idiopathic disorders. Medicine (Baltimore) 63: 343-361

27. de Prost N, Parrot A, Picard C, Ancel PY, Mayaud C, Fartoukh M, Cadranel J, (2010) Diffuse alveolar haemorrhage: factors associated with in-hospital and long-term mortality. Eur Respir J 35: 1303-1311

28. de Prost N, Parrot A, Cuquemelle E, Picard C, Antoine M, Fleury-Feith J, Mayaud C, Boffa JJ, Fartoukh M, Cadranel J, (2012) Diffuse alveolar hemorrhage in immunocompetent patients: etiologies and prognosis revisited. Respir Med 106: 1021-1032

29. Cormier Y, Israël-Assayag E, (2000) The role of viruses in the pathogenesis of hypersensitivity pneumonitis. Curr Opin Pulm Med 6: 420-423

30. Girard M, Lacasse Y, Cormier Y, (2009) Hypersensitivity pneumonitis. Allergy 64: 322-334 
31. Ho D, Tashkin DP, Bein ME, Sharma O, (1979) Pulmonary infiltrates with eosinophilia associated with tetracycline. Chest 76: $33-36$

32. Philit F, Etienne-Mastroïanni B, Parrot A, Guérin C, Robert D, Cordier JF, (2002) Idiopathic acute eosinophilic pneumonia: a study of 22 patients. Am J Respir Crit Care Med 166: 1235-1239

33. Guérin C, Thompson T, Brower R, (2015) The ten diseases that look like ARDS. Intensive Care Med 41: 1099-1102

34. Papazian L, Doddoli C, Chetaille B, Gernez Y, Thirion X, Roch A, Donati Y, Bonnety M, Zandotti C, Thomas P, (2007) A contributive result of open-lung biopsy improves survival in acute respiratory distress syndrome patients: Crit Care Med 35: 755-762

35. Thompson BT, Guérin C, Esteban A, (2016) Should ARDS be renamed diffuse alveolar damage? Intensive Care Med 42: 653-655

36. Gajic O, Dabbagh O, Park PK, Adesanya A, Chang SY, Hou P, Anderson H 3rd, Hoth JJ, Mikkelsen ME, Gentile NT, Gong MN, Talmor D, Bajwa E, Watkins TR, Festic E, Yilmaz M, Iscimen R, Kaufman DA, Esper AM, Sadikot R, Douglas I, Sevransky J, Malinchoc M; U.S. Critical Illness and Injury Trials Group: Lung Injury Prevention Study Investigators (USCIITG-LIPS), (2011) Early identification of patients at risk of acute lung injury: evaluation of lung injury prediction score in a multicenter cohort study. Am J Respir Crit Care Med 183: 462-470

37. de Prost N, Ricard JD, Saumon G, Dreyfuss D, (2011) Ventilatorinduced lung injury: historical perspectives and clinical implications. Ann Intensive Care 1: 28

38. Schwarz MI, Albert RK, (2004) "Imitators" of the ARDS: implications for diagnosis and treatment. Chest 125: 1530-1535

39. Papazian L, Calfee CS, Chiumello D, Luyt CE, Meyer NJ, Sekiguchi H, Matthay MA, Meduri GU, (2016) Diagnostic workup for ARDS patients. Intensive Care Med 42: 674-685
40. Choi SH, Hong SB, Ko GB, Lee Y, Park HJ, Park SY, Moon SM, Cho OH, Park KH, Chong YP, Kim SH, Huh JW, Sung H, Do KH, Lee SO, Kim MN, Jeong JY, Lim CM, Kim YS, Woo JH, Koh Y, (2012) Viral infection in patients with severe pneumonia requiring intensive care unit admission. Am J Respir Crit Care Med 186: 325-332

41. Palakshappa JA, Meyer NJ, (2015) Which patients with ARDS benefit from lung biopsy? Chest 148: 1073-1082

42. Forel JM, Guervilly C, Hraiech S, Voillet F, Thomas G, Somma C, Secq V, Farnarier C, Payan MJ, Donati SY, Perrin G, Trousse D, Dizier S, Chiche L, Baumstarck K, Roch A, Papazian L, (2015) Type III procollagen is a reliable marker of ARDSassociated lung fibroproliferation. Intensive Care Med 41: 1-11

43. Papazian L, Gainnier M, (2003) Indications of BAL, lung biopsy, or both in mechanically ventilated patients with unexplained infiltrations. Eur Respir J 21: 383-384

44. Guérin C, Reignier J, Richard JC, Beuret P, Gacouin A, Boulain T, Mercier E, Badet M, Mercat A, Baudin O, Clavel M, Chatellier D, Jaber S, Rosselli S, Mancebo J, Sirodot M, Hilbert G, Bengler C, Richecoeur J, Gainnier M, Bayle F, Bourdin G, Leray V, Girard R, Baboi L, Ayzac L; PROSEVA Study Group, (2013) Prone positioning in severe acute respiratory distress syndrome. N Engl J Med 368: 2159-2168

45. Papazian L, Forel JM, Gacouin A, Penot-Ragon C, Perrin G, Loundou A, Jaber S, Arnal JM, Perez D, Seghboyan JM, Constantin JM, Courant P, Lefrant JY, Guérin C, Prat G, Morange S, Roch A; ACURASYS Study Investigators, (2010) Neuromuscular blockers in early acute respiratory distress syndrome. N Engl J Med 363: 1107-1116 